Z. klin. Chem. u. klin. Biochem.

9. Jg., S. 245-248, Mai 1971

\title{
Experimentelle Myotonie durch 20.25-Diazacholesterin
}

\section{- Analyse der Phospholipide und Cholesterinester des sarkoplasmatischen Retikulum und des Sarkolemm -}

Von D. SeILER und E. KuHN

Medizinische Poliklinik (Kommiss. Direktor: Prof. Dr. E. Kubn) der Universität Heidelberg

(Eingegangen am 8. Februar 1971)

\begin{abstract}
Aus der Skelettmuskulatur von Ratten mit Myotonie durch 20.25-Diazacholesterin und aus der von Kontrollratten wurden die Vesikel des sarkoplasmatischen Retikulum und des Sarkolemm isoliert und die Fettsäurezusammensetzung der Phospholipide dieser Fraktionen bestimmt. Bei den mit 20.25-Diazacholesterin behandelten Ratten wurde im Vergleich zu den Kontrollratten eine Zunahme der Stearin-, der Arachidon- und der Dokosahexaensäure, sowie eine Abnahme der Palmitin- und der Olsäure in beiden Membranfraktionen gefunden.

Das Fettsäuremuster der Cholesterinester dieser Fraktionen war in der gleichen Weise verändert.

Die Phospholipidzusammensetzung der sarkoplasmatischen Vesikel und des nach Isolierung der Vesikel zurückbleibenden Muskels zeigte keine Unterschiede zwischen normalen und myotonen Ratten.

Der Gesamtlipidgehalt der sarkoplasmatischen Vesikel von normalen und myotonen Ratten war glcich.
\end{abstract}

\section{Experimental myotonia with 20.25-diazacholesterol - Analysis of the phospholipids and cholesterol esters of the Sarcoplasmic} reticulum and the sarcolemma

The fatty acid composition of the phospholipids from sarcoplasmic vesicles and sarcolemma from the skeletal muscles of normal rats and of rats with myotonia induced by treatment with 20.25-diazacholesterol were compared. In 20.25-diazacholesterol-treated rats there was an increase in stearic-, arachidonic-, and docosahexaenoic acid and a decrease in palmitic-, and oleic acid in both membrane fractions. The fatty acid pattern of the cholesterol esters of these fractions were altered in the same way.

The phospholipid composition of the sarcoplasmic vesicles and of the muscle remaining after isolation of these vesicles shows no differences between normal and myotonic rats.

The total lipid content of the sarcoplasmic vesicles from normal and myotonic rats proved to be equivalent.

Die durch längerdauernde Gabe von 20.25-Diazacholesterin induzierte Myotonie läßt sich elektromyographisch und mechanographisch von der hereditären Myotonie des Menschen nicht unterscheiden (1), und auch bei der Ratte treten nach längerdauernder Sondenfütterung die Symptome der Myotonie auf. Das Fettsäuremuster der Phospholipide aus der unfraktionierten Skelettmuskulatur von Ratten, die mit 20.25-Diazacholesterin behandelt wurden, unterscheidet sich eindeutig von dem der unbehandelten Ratten (2). Ähnliche Befunde erhielten wir bei Ratten mit amin-induzierter Myotonie (3), und auch bei Patienten mit Myotonia congenita fanden wir Veränderungen im Fettsäuremuster der Muskelphospholipide gegenüber Muskelgesunden (4).

Der Einbau von 20.25-Diazadehydrocholesterinestern in die Vesikel des sarkoplasmatischen Retikulum (5) und Veränderungen des Calciumtransportsystems dieser Vesikel (G) bei Ratten mit durch 20.25-Diazacholesterin induzierter Myotonie wurden beschrieben. Patienten mit Myotonia congenita und Myotonia dystrophica zeigten ebenfalls Unterschiede in der Calcium-Transportfähigkeit der Vesikel des sarkoplasmatischen Retikulum (7):

$\mathrm{Da}$ die Myotonie, wie heute allgemein angenommen wird, ein Membranphänomen zu sein scheint und die bisherigen Fettsäureanalysen der Phospholipide nur an der Gesamtmuskulatur durchgeführt wurden, versuchten wir festzustellen, ob die Unterschiede in der
Rattenmuskulatur auch in den für die Entstehung der Myotonie hauptsächlich in Frage kommenden Membransystemen des sarkoplasmatischen Retikulum und des Sarkolemm zu finden sind.

Veränderungen der Fettsäurezusammensetzung könnten durch Änderung des Verhältnisses der Einzelphospholipide bewirkt werden; deshalb untersuchten wir auch die Phospholipidzusammensetzung des Muskels und des isolierten sarkoplasmatischen Retikulum.

Weiterhin analysierten wir die Fettsäurezusammensetzung der für die Funktion von Membranen ebenfalls wichtigen Cholesterinester des sarkoplasmatischen Retikulum und des Sarkolemm.

\section{Methodik}

Weiblichen Wistar-Ratten mit einem Körpergewicht von 200 bis $250 \mathrm{~g}$ wurde $6-8$ Wochen lang $6 \mathrm{mal}$ pro Woche $10 \mathrm{mg}$ 20.25-Diazacholesterindihydrochlorid in $1 \mathrm{~m} l$ Wasser mit einer Magensonde gegeben. Die Gesamtdosis betrug 360-480 mg pro Tier.

Die als Kontrollen gehaltenen gleichaltrigen Tiere erhielten im gleichen Zeitraum jeweils $1 \mathrm{~m} l$ Wasser, $6 \mathrm{mal}$ pro Woche, mit der Magensonde. Alle Tiere bekamen täglich $12-14 \mathrm{~g}$ StandardFutter der Firma Latz, Euskirchen und Wasser ad libitum. In der Nahrungsaufnahme konnten keine Unterschiede zwischen den mit 20.25-Diazacholesterin behandelten und den unbehandelten Tieren festgestellt werden und die Gewichtszunahme verlief bei beiden Gruppen im Behandlungszeitraum ungefähr gleich.

Die Isolierung der Vesikel des sarkoplasmatischen Retikulum erfolgte nach HAsselbacir und MAakinose (8), die des Sarkolemm 
nach RosenthaL und Mitarbeitern (9) aus dex Skelettmuskulatur der Ratten.

Die Vesikel des sarkoplasmatischen Retikulum waren elektronenmikroskopisch rein, während die Sarkolemmfraktion noch durch Myofibrillen verunreinigt war.

Die isolierten Membranfraktionen wurden mit 20 Teilen Chloroform/Methanol $(2: 1 ; \nabla / v) 24$ Stdn. unter Stickstoff bei Raumtemperatur extrahiert und die filtrierte Lösung nach SPERRY (10) gereinigt. Die Phospholipide wurden chromatographisch von den Neutrallipiden getrennt (11), mit $2 \mathrm{~N}$ methanol. $\mathrm{KOH}$ verseift und anschließend mit 14proz. methanol. Bortrifluoridlösung verestert (12). Die Cholesterinester wurden dünnschichtchromatographisch von den übrigen Neutrallipiden abgetrennt (13) und mit 5proz. methanol. Salzsäure unter Zusatz von Essigsäuremethylester (14) umgeestert. Die Reinheit der Phospholipid- und der Cholesterinesterfraktion wurde dünnschichtchromatographisch überprüft.

Gaschromatographie der Fettsäuremethylester: Gaschromatograph Varian aerograph 1840-3, Flammenionisationsdetektor, Säule $10^{\prime} \times 1 / 8^{\prime \prime} \mathrm{mit} 5 \%$ EGSS-X auf Chromosorb WAW 80-100 mesh; $36 \mathrm{ml} \mathrm{N} /$ Min.; $180^{\circ}$ isotherm.

Die Peaks wurden durch Standards identifiziert oder durch Vergleich mit den Fettsäuren aus Bullentestes (15) bestimmt. Die Auftrennung und quantitative Bestimmung der Phospholipide führten wir nach OwENs (16) durch.

Der Lipidgehalt der sarkoplasmatischen Vesikel wurde durch Extraktion der mit Wasser gewaschenen, gefriergetrockneten und gewogenen Vesikel mit Chloroform/Methanol $(2: 1 ; \mathrm{v} / \mathrm{v})$ oder Diäthyläther/A thanol $(3: 1 ; \nabla / v)$ und Auswiegen des abzentrifugierten und eingedampften Extraktes bestimmt. Als Kontrolle wogen wir auch den getrockneten Eiweißrückstand.

\section{Ergebnisse}

\section{Fettsäuremuster der Phospholipide der Vesikel des sarko-} plasmatischen Retikulum

In der Tabelle 1 sind die relativen Prozentzahlen der Fettsäuren, bei denen wir Veränderungen fanden, aufgeführt. Es handelt sich um Palmitinsäure $\left(C_{16: 0}\right)$,
Stearinsäure $\left(\mathrm{C}_{18: 0}\right)$, Ölsäure $\left(\mathrm{C}_{18: 1}\right)$, Atachidonsäure $\left(C_{20: 4}\right)$ und um Dokosahexaensäure $\left(C_{22: 6}\right)$. Die anderen Fettsäuren der Phospholipide und die aus den Plasmalogenen entstandenen Dimethylacetale waren unverändert.

\section{Fettsäuremuster der Pbospbolipide des Sarkolemm}

Die Veränderungen der Fettşäurezusammensetzung betreffen hier wieder die gleichen Säuren wie bei den Vesikeln des sarkoplasmatischen Retikulum. In Tabelle 2 sind die relativen Prozentzahlen der veränderten Säuren angegeben. Die Dokosahexaensäure konnten wir weder im Sarkolemm der Kontrolltiere noch in dem der myotonen Tiere nachweisen.

\section{Fettsäuremuster der Cholesterinester der sarkoplasmatischen Vesikel und des Sarkolemm}

Auch hier finden sich wieder Veränderungen der Palmitinsäure, der Ölsäure und der Arachidonsäure. Dokosahexaensäure konnten wir, im Gegensatz zu den von Fremn und Hasselbach (17) bei Kaninchenvesikeln erhaltenen Ergebnissen, in keiner Präparation aus Rattenmuskulatur nachweisen. Die Stẹarinsäure zeigt weder bei den Cholesterinestern der sarkoplasmatischen Vesikel noch bei denen des Sarkolemm Unterschiede zwischen Kontrolltieren und myotonen Tieren. Tabelle 3 gibt die relativen Prozentzahlen der veränderten Fettsäuren an.

\section{Zusammensetzung der Phospholipide}

Die Zusammensetzung der Phospholipide wurde in den Vesikeln des sarkoplasmatischen Retikulum und in dem nach Abtrennung der Vesikel verbleibenden Rest-

Tab. 1

Fettsäurezusammensetzung der Phospholipide der Vesikel des sarkoplasmatischen Retikulum (in \%; Summe aller Fettsäurepeaks $=100 \%$ ). Mittelwerte aus 10 Bestimmungen in jeder Gruppe. Die Zahlen in Klammern geben die gefundenen Extremwerte an

\begin{tabular}{|c|c|c|c|c|c|}
\hline Fettsäure & $\mathrm{c}_{16: 0}$ & $C_{18: 0}$ & $C_{18: 1}$ & $\mathrm{C}_{20 ; 4}$ & $C_{22: 6}$ \\
\hline $\begin{array}{l}\text { Kontroll-Ratten } \\
\text { Myotone Ratten }\end{array}$ & $\begin{array}{c}43,1 \\
(39,5-48,4) \\
35,4 \\
(31,5-37,6)\end{array}$ & $\begin{array}{c}7,3 \\
(5,8-10,9) \\
12,2 \\
(10,7-14,1)\end{array}$ & $\begin{array}{c}24,4 \\
(17,2-29,8) \\
15,0 \\
(10,2-19,7)\end{array}$ & $\begin{array}{c}(1,8,5 \\
10,9,5) \\
(8,5-12,4)\end{array}$ & $\begin{array}{c}4,4 \\
(3,0-5,9) \\
7,8 \\
(6,8-13,6)\end{array}$ \\
\hline
\end{tabular}

Tab. 2

Fettsäurezusammensetzung der Phospholipide des Sarkolemm (in \%; Summe der Fettsäurepeaks $=100 \%$ ). Mittelwerte aus je 5 Bestimmungen. (Die Sarkolemmfraktionen von jeweils 2 Tieren wurden wegen der geringen Menge zusammen extrahiert.) Die Zahlen in Klammern geben die gefundenen Extremwerte an

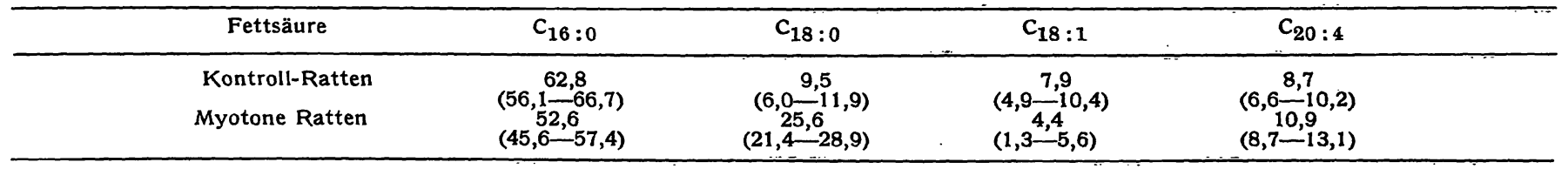

Tab. 3

Fettsäurezusammensetzung der Cholesterinester des sarkoplasmatischen Retikulum und des Sarkolemm. (In \%; Summe der Fettsäurepeaks $=100 \%$.) Mittelwerte aus je 4 Bestimmungen. (Wegen der geringen Menge wurden immer die Cholesterinester aus Sarkolemm bzw. sarkoplasmatischem Retikulum von 5 Ratten gepoolt.) Die Zahlen in Klammern geben die gefundenen Extremwerte an

\begin{tabular}{|c|c|c|c|}
\hline Fettsäure & $C_{16: 0}$ & $c_{18: 1}$ & $C_{20: 4}$ \\
\hline $\begin{array}{l}\text { Sarkoplasmatisches Retikulum von Kontrollratten } \\
\text { Sarkoplasmatisches Retikulum von myotonen Ratten } \\
\text { Sarkolemm von Kontrollratten } \\
\text { Sarkolemm von myotonen Ratten }\end{array}$ & $\begin{array}{l}37,8 \\
(36,1-39,5) \\
(33,0,0,35,6) \\
(33,7-3-39,3) \\
(30,94-36,5)\end{array}$ & $\begin{array}{l}34,8 \\
(33,2-35,4) \\
(20,2-2,-26,1) \\
(28,0-34,7) \\
(24,9-28,0,4)\end{array}$ & $\begin{array}{l}2,0 \\
(0,5-3,0) \\
\left(5,8-\frac{8,3}{3,0,0)}\right. \\
(1,2-5,4) \\
(6,3-11,7)\end{array}$ \\
\hline
\end{tabular}


Tab. 4

Zusammensetzung der Phospholipidfraktion (als \% der Gesamtphospholipide). Mittelwerte aus jeweils 10 Bestimmungen mit 10 Versuchstieren in jeder Gruppe. Die Zahlen in Klammern geben die gefundenen Extremwerte an

\begin{tabular}{|c|c|c|c|c|}
\hline & \multicolumn{2}{|c|}{ Restmuskel } & \multicolumn{2}{|c|}{ Sarkoplasmatische Vesikel } \\
\hline & Kontrollratten & Myotone Ratten & Kontrollratten & Myotone Ratten \\
\hline $\begin{array}{l}\text { Lyso-phosphatidylcholin } \\
\text { Sphingomyelin } \\
\text { Phosphatidylcholin } \\
\text { Cholin-Plasmalogen } \\
\text { Phosphatidylserin + Phosphatidylinosit } \\
\text { Phosphatidyläthanolamin } \\
\text { Äthanolamin-Plasmalogen } \\
\text { "Nicht identifizierte Phospholipide (Cardiolipin?)“ }\end{array}$ & $\begin{array}{c}\left(\frac{-}{3,5}\right) \\
\left(2,3 \frac{4,7}{47,2}\right. \\
\left(41,5 \frac{51,9}{4,7}\right) \\
\left(2,5 \frac{-8,3)}{7,1}\right. \\
\left(5,8 \frac{10,1)}{16,7}\right. \\
(15,0-19,1) \\
\left(10,7 \frac{12,6}{4,8}\right) \\
(1,3-7,1)\end{array}$ & $\begin{array}{c}\left(\frac{-}{5,5}\right) \\
\left(4,3 \frac{3,6,9)}{44,1}\right. \\
\left(41,3 \frac{48,4)}{3,4}\right. \\
\left(2,5 \frac{4,0)}{8,7}\right. \\
\left(5,2 \frac{1}{17} 0,4\right) \\
\left(14,7 \frac{1}{3,0}, 9,7\right) \\
\left(10,8 \frac{1}{5,2}\right) \\
(4,4-6,2)\end{array}$ & $\begin{array}{c}\left(0,2 \frac{1,2}{3,2}, 2\right) \\
\left(2,6 \frac{4,3)}{63,8}\right. \\
\left(57,5 \frac{-71}{5,6}\right) \\
(3,5-7,7) \\
\left(8,5 \frac{10,0}{9,6} 1,5\right) \\
\left(7,8 \frac{-12,3)}{5,4}\right. \\
\left(3,9 \frac{6,7)}{1,2}\right. \\
(0,9-1,7)\end{array}$ & $\begin{array}{c}\left(0,1 \frac{1,3}{3,2,3)}\right. \\
\left(2,3 \frac{3,9}{6,7}\right) \\
\left(50,1 \frac{64,6}{5,3}, 5\right) \\
\left(3,7 \frac{-7,3)}{9,0}\right. \\
\left(5,9 \frac{1}{9,4} 0,9\right) \\
\left(7,6 \frac{1}{4,6} 1,0\right) \\
\left(2,4 \frac{-8,1)}{1,4}\right. \\
(0,5-2,5)\end{array}$ \\
\hline
\end{tabular}

muskel bestimmt. Die Ergebnisse sind in Tabelle 4 dargestellt. Phosphatidylserin und Phosphatidylinosit konnten nicht immer eindeutig getrennt werden und wurden deshalb zusammen bestimmt. Mit „nicht identifizierte Phospholipide" ist der nicht eindeutig identifizierte, wahrscheinlich Cardiolipin enthaltende Fleck an der Front des Dünnschichtchromatogramms nach dem ersten Lauf bezeichnet. Im Restmuskel fanden wir kein Lysophosphatidylcholin und es ist sehr wahrscheinlich, daß das in den Vesikeln des sarkoplasmatischen Retikulum gefundene Lysophosphatidylcholin ein durch die längere Aufarbeitung bedingtes Kunstprodukt darstellt.

\section{Lipidgebalt der Vesikel des sarkoplasmatischen Retikulum}

Der Lipidgehalt der Vesikel des sarkoplasmatischen Retikulum von myotonen und Kontrollratten unterscheidet sich nicht. Wir fanden für die Vesikel von myotonen Ratten einen Lipidgehalt von $59,8 \mathrm{mg}$ pro $100 \mathrm{mg}$ Vesikelprotein (Extremwerte bei 10 Versuchen: 56,3-62,3) und für die der Kontrollratten $59,9 \mathrm{mg}$ Lipide pro $100 \mathrm{mg}$ Vesikelprotein (Extremwerte bei 10 Versuchen: 54,0-62,8). Diese Ergebnisse stimmen mit den von DraBIKowskI und Mitarbeitern (18) und von BALzer und Mitarbeitern (19) für Vesikel des sarkoplasmatischen Retikulum aus Kaninchenmuskulatur erhaltenen Werten überein.

\section{Diskussion}

Beim Vergleich der Fettsäureveränderungen der Phospholipide des sarkoplasmatischen Retikulum und des Sarkolemm mit den früher (2) gefundenen Veränderungen der Fettsäuren der Gesamtmuskelphospholipide sieht man, daß die Veränderungen im wesentlichen immer die gleichen Säuren betreffen und auch alle in der gleichen Richtung verlaufen. Wir fanden bei myotonen Ratten eine Abnahme der Palmitin- und der Ölsäure und eine Zunahme der Stearin- und Arachidonsäure. Die Dokosahexaensäure konnte im Sarkolemm nicht nachgewiesen werden, ist aber im sarkoplasmatischen Retikulum und im Gesamtmuskel der myotonen Ratten vermehrt. Die Zunahme der Arachidonsäure könnte zum Teil bedingt sein durch das Auf- treten von 20.25-Diazadehydrocholesterinestern, bei denen wir einen Arachidonsäuregehalt von 37\% gefunden haben (5). Diese Ester, die ungefähr 1-2\% der in den Vesikeln des sarkoplasmatischen Retikulum und im Sarkolemm enthaltenen Lipide ausmachen, verbleiben in dem angewandten Trennungsgang bei den Phospholipiden.

Betrachtet man die Veränderungen in der Zusammensetzung der - prozentual in den untersuchten Fraktionen in sehr viel geringerer Menge vorkommenden Cholesterinester, so kann man feststellen, daß auch hier eine Verminderung der Palmitin- und der Ölsäure und eine Erhöhung der Arachidonsäure bei myotonen Ratten auftreten. Die Stearinsäure, die in den Cholesterinestern der sarkoplasmatischen Vesikel zu 13\% und in denen das Sarkolemm zu 15\% enthalten ist, ist beim Vergleich von Kontrollratten und myotonen Ratten unverändert. $\mathrm{Da}$ die Fraktion der Cholesterinester frei von Estern des 20.25-Diazadehydrocholesterin ist und die einzelnen Cholesterinester nur durch die mit ihnen veresterten Fettsäuren unterschiedlich sind, handelt es sich bei den gefundenen Veränderungen um echte Veränderungen des Fettsäuremusters, während die differenten Fettsäuremuster der Phospholipide auch durch einen unterschiedlichen Gehalt an Einzelphospholipiden hervorgerufen werden könnten.

Bei dystrophischen Mäusen (20) und bei Patienten mit progressiver Muskeldystrophie (21) sind Unterschiede in der Phospholipidzusammensetzung im Sinne einer Erniedrigung der Fraktion Phosphatidylcholin + Cholinplasmalogen und einer Erhöhung des Sphingomyelins nachgewiesen worden. Diese Unterschiede waren bei den Mäusen besonders deutlich in der $35000 \mathrm{~g}$-Fraktion, die einem unreinen sarkoplasmatischen Retikulum entspricht (22).

Unsere Ergebnisse zeigen, daß bei Ratten mit durch 20.25-Diazacholesterin induzierter Myotonie die Phospholipidzusammensetzung der Vesikel des sarkoplasmatischen Retikulum im Vergleich zu den Vesikeln der Kontrollratten unverändert ist. Auch in der Phospholipidzusammensetzung des Restmuskels finden sich keine eindeutigen Unterschiede, die die Veränderungen im Fettsäuremuster der Phospholipide erklären könnten. 
Die Frage einer möglichen Veränderung des Gesamtlipidgehaltes prüften wir an der am reinsten zu gewinnenden Fraktion, den Vesikeln des sarkoplasmatischen Retikulum. Wir fanden aber keine Unterschiede im Lipidgehalt der Vesikel von Kontrollratten und myotonen Ratten.

Die Tatsache, daß sowohl im Sarkolemm als auch im sarkoplasmatischen Retikulum gleichartige Unterschiede angetroffen wurden, spricht für eine allgemeine Fettsäureveränderung der Membranen unter dem Einfluß von 20.25-Diazacholesterin. Diese Úberlegung wird unterstützt durch den Befund, daß auch in den Cholesterinestern von Sarkolemm und sarkoplasmati- schen Vesikeln ähnliche Fettsäureveränderungen bewirkt werden.

$\mathrm{Ob}$ die in der Gesamtmuskulatur von Patienten mit Myotonia congenita gefundenen Unterschiede im Fettsäuremuster der Phospholipide (7) ebenfalls mehrere Membranen betreffen und nicht durch veränderten Gehalt an Einzelphospholipiden bedingt sind, muß in weiteren Untersuchungen geklärt werden.

Diese Arbeit wurde mit Unterstützung durch die Deutsche Forschungsgemeinschaft, Bad Godesberg, durchgeführt.

Wir danken Frl. I. Geldmacher, Frl. R. Kipphan und Frl E. Threle für ihre Mitarbeit bei der Durchführung der Bestimmungen.

\section{Literatur}

1. Winer, N., J. M. MArtt, J. E. Somers, L. Wolcotr, H. E. Dale und T. W. Burns, J. Laborat. Clin. Med., S. Louis 66, 758 (1965). - 2. KuhN, E., W. Dorow, W. Kahlke und H. Pfisterę, Klin. Wschr. 46F, 1043 (1968). - 3. SeILER, D. und E. KuHN, Klin. Wschr. 47, 1114 (1969). - 4. KunN, E. und D. SEILER, Klin. Wschr. 48, 1134 (1970). - 5. SeIler, D. und E. KuHN, Eur. J. Biochem. 11, 175 (1969). - 6. SeIleR, D., E. KunN, W. Fiefn und W. Hasselbach, Eur. J. Biochem. 12, 375 (1970). 7. SeIler, D. ind E. KunN, Schweiz. med. Wschr. 100, 1374 (1970). - 8. Hasselbach, W. und M. Makinose, Biochem. Z. 339, 94 (1963). - 9. Rosenthal, S. L., P. M. Edelman und J. L. SchwarTz, Biochim. biophys. Acta (Amsterdam) 109, 512 (1965). 10. SPERRY, W. M., Meth. biochem. Anal. 2, 83 (1955). - 11. Borgström, B., Acta physiol. Scand. 25, 101 (1952). - 12 Metcalfe, L. D. und A. A. Schmrtz, Analytic. Chem. 33, 363 (1961). - 13. SKIPSKI, V. P. und M. BARCLEY, In: Methods in
Enzymology, Vol. 14, S. 549, J. M. Lowenstein (ed.) Academic Press. N. Y. u. London (1969). - 14. Eberhagen, D., HoppeSeylers, Z. physiol. Chem. 333, 179 (1963). - 15. Holman, R. T. und J. J. Rarm, Progress Chem. Fats Lipids 9 part 1, 52 (1966). 16. Owens, K., Biochem. J. 100, 354 (1966). - 17. FIEHN, W. und W. Hasselbach, Eur. J. Biochem. 9, 574 (1969). - 18. Drabikowskr, W., H. Domnas und M. Dabrowska, Acta biochem. polon. 13, 12 (1966). - 19. BALzER, H., M. MAKINose, W. FieHN und W. HASSELBACH, Naunyn-Schmiedebergs Atch. exper. Path. 260, 456 (1969). - 20. Hughes, B. P. und F. F. Frats, Biochem. J. 96, 6P (1965). - 21. Kunze, D., D. OlthofF und K. Schellnack, Acta biol. med. germ. 19, 1057 (1967). 22. Hughes, B. P., in: Research in Muscular Dystrophie. Proceedings of the 4th Symposium, S. 286. Pitman Medical Publishing, London (1968).
Dieter Seiler

Med. Poliklinik der Universität 6900 Heidelberg Hospitalstraße 3 


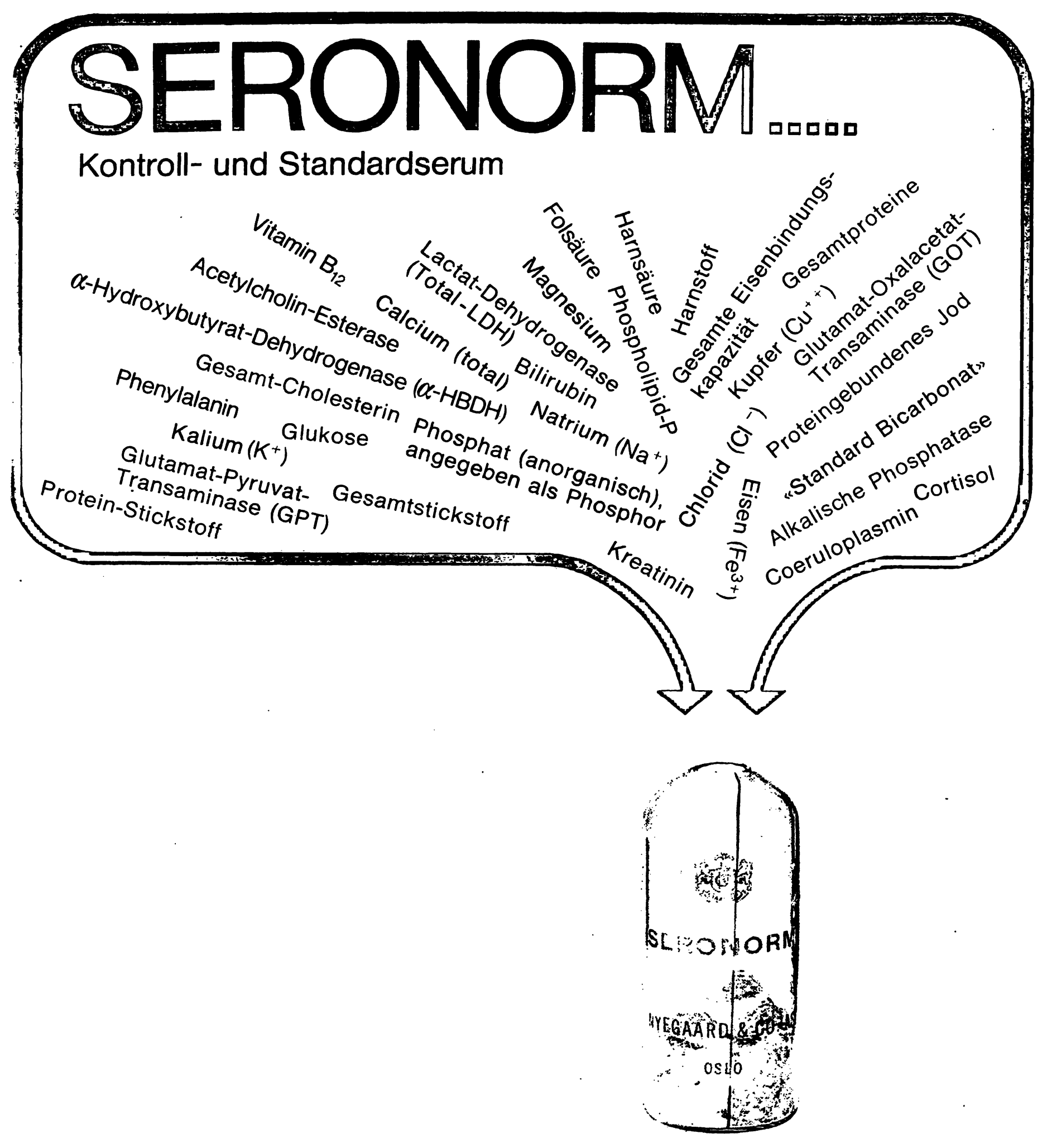

\section{SERONORM}

enthält 32 analysierte Serumkomponenten, deren empfohlene Werte in 5 anerkannten Laboratorien nach verschiedenen modernen Methoden festgelegt sind.

\section{SERONORM}

ist in der verschlossenen VakuumAmpulle 4 Jahre haltbar und in Packungen zu $5 \times 10 \mathrm{ml}$ erhältlich.

\section{SERONORM}

gehört auch in Ihr Laboratorium, denn es ermöglicht Ihnen die laufende Überwachung Ihrer Analysenwerte.

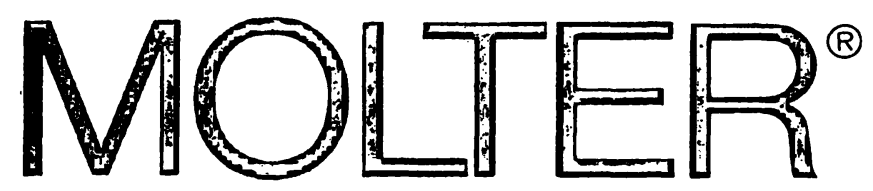

Dr. Molter $\mathrm{GmbH}$, Serum-Institut, 69 Heidelberg 1 Postfach 1210. Telefon 06223/5337 


\section{SORVALL-Zentrifugen gibt es viele...}

Hochgeschwindigkeit, g-Werte, Kühlung, Automatik,

Durchfluß-System, Spezial-Rotore, Blutaufbereitung - das sind nur einige Begriffe.

\section{Am besten, Sie nennen uns das * Problem}

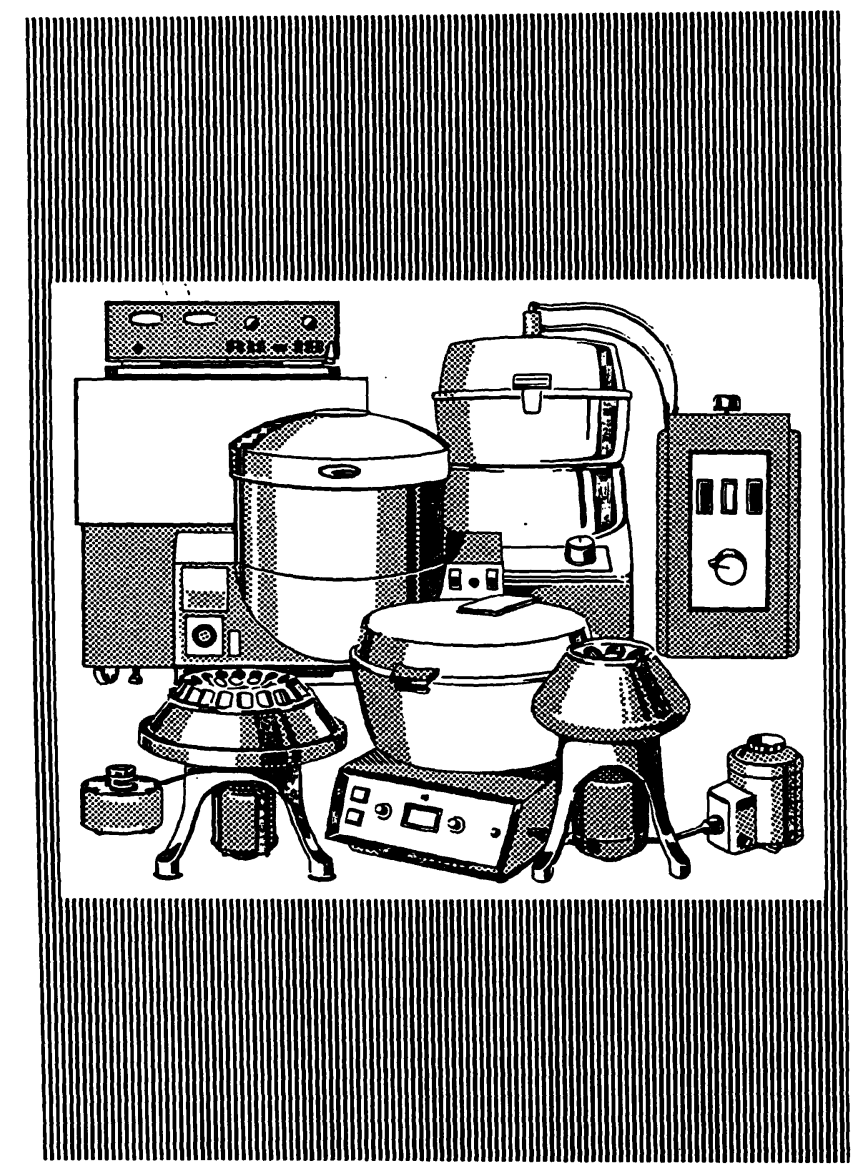

* damit Sie gleich die spezielle Druckschrift bekommen.

\section{Unsere interessanten Druckschriften SOR-Z informieren Sie ausführlich.}

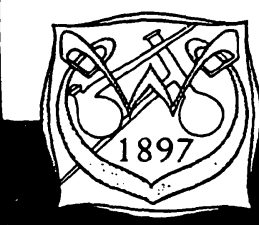

WISSENSCHAFTLICHE APPARATE

\section{HORMUTH-VETTER}

6908 Wiesloch/Bd., Postfach 1348, Tel. $06222 / 2147$ 6900 Heidelberg 1, Postfach 750, Tel. 0622120045
Helmut A. Fischer - Gottfried Werner

\section{Autoradiographie}

Oktav. IX, 214 Seiten. Mit 93 Abbildungen und 14 Tabellen. 1971. Werkstoff DM 42,-

In den letzten Jahren hat die Methodik der Autoradiographie in zahlreichen naturwissenschaftlichen Forschungsgebieten Anwendung gefunden. In dem vorliegenden Buche „Autoradiographie“ werden - nach einer kurz gehaltenen Einleitung über die allgemeinen physikalischen Grundlagen - erstmals alle wesentlichen Techniken für die verschiedenen Anwendungsgebiete beschrieben, typische Beispiele aus den betreffenden Fachgebieten (z. B. Biologie, alle Gebiete der Medizin, Chemie, Physik, Biochemie, Kriminalistik, Pharmakologie und Toxikologie) gegeben und die Möglichkeiten und Grenzen der qualitativen und quantitativen Autoradiographie kritisch behandelt. Die Gliederung des Stoffes nach der Dimension (makroskopisch, mikroskopisch und clektronenmikroskopisch) der autoradiographisch untersuchten Objekte ergibt eine gute Übersichtlichkeit der sehr differenten Anwendungsgebiete. Die, relativ zur verarbeiteten Gesamtliteratur von etwa 10000 Literaturzitaten, geringe Zahl von 500 textbezogenen Literaturangaben soll das Auffinden der in den verschiedenen Zeitschriften weit gestreuten Publikationen erleichtern.

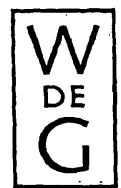

Walter de Gruyter Berlin - New York 